\title{
Outcomes From Treatment of Infertility With Natural Procreative Technology in an Irish General Practice
}

\author{
Joseph B. Stanford, MD, MSPH, Tracey A. Parnell, MD, and Phil C. Boyle, MB
}

Objectives: We evaluated outcomes in couples treated for infertility with natural procreative technology (NaProTechnology, NPT), a systematic medical approach for optimizing physiologic conditions for conception in vivo, from an Irish general practice.

Methods: All couples receiving treatment from 2 NPT-trained family physicians between February 1998 and January 2002 were studied. The main outcome was live birth, and secondary outcomes included conceptions and multiple births. Crude proportions and adjusted life-table proportions were calculated per 100 couples.

Results: A total of 1239 couples had an initial consult for NPT, of which 1072 had been trying for at least a year to conceive and initiated treatment. The average female age was 35.8 years, the mean duration of attempting to conceive was 5.6 years, $24 \%$ had a prior birth, and $33 \%$ had previously attempted treatment with assisted reproductive technology (ART). All couples were taught to identify the fertile days of the menstrual cycle with the Creighton Model FertilityCare System, and most received additional medical treatment, including clomiphene (75\%). In life-table analysis, the cumulative proportion of first live births for those completing up to 24 months of NPT treatment was 52.8 per 100 couples. The crude proportion was 25.5. Younger couples and couples without previous ART attempts had higher rates of live birth. Among live births, there were $4.6 \%$ twin births.

Conclusion: NPT provided by trained general practitioners had live birth rates comparable to cohort studies of more invasive treatments, including ART. Further studies are warranted to compare NPT directly to other treatments. (J Am Board Fam Med 2008;21:375-384.)

Infertility is a common problem; one in every 7 couples hoping to have a baby experiences difficulties achieving or maintaining pregnancy serious enough to seek medical intervention. ${ }^{1}$ Infertility is a chronic problem that involves both women and

This article was externally peer reviewed.

Submitted 30 October 2007; revised 11 April 2008; accepted 15 April 2008.

From the Department of Family and Preventive Medicine, University of Utah, Salt Lake City (JBS); the Department of Family Medicine, University of British Columbia, Vancouver, Canada (TAP); the Galway Clinic, Ireland (PCB); and the International Institute of Restorative Reproductive Medicine, London, United Kingdom (JBS, TAP, PCB).

Funding: none.

Prior presentation: Portions of this work have been presented at the North American Primary Care Research Conference, Vancouver, Canada, 21 October 2007.

Conflict of interest: none declared.

Corresponding author: Dr. Joseph B. Stanford, MD, MSPH, University of Utah, Department of Family and Preventive Medicine, 375 Chipeta Way, Suite A, Salt Lake City, UT 84108 (E-mail: joseph.stanford@utah.edu). men, has major psychosocial ramifications, and usually requires addressing multiple issues, including comorbid medical conditions and lifestyle, all key elements of primary care practice. However, treatments for infertility are largely provided by physicians trained in subspecialties, including artificial insemination, ovulation induction, and hormonal support of the menstrual cycle. ${ }^{1,2}$ The increasing shift of treatment toward assisted reproductive technologies (ART), including in vitro fertilization and intracytoplasmic sperm injection, has removed infertility treatment further from the realm of the generalist or family physician. ${ }^{1,3}$

ART is expensive, invasive, and involves some risk to women. These include risks of the medical and surgical procedures to retrieve oocytes, ${ }^{4}$ including ovarian hyperstimulation syndrome. ${ }^{5}$ There are also concerns about short- and longterm outcomes for the offspring. ${ }^{6-9}$ The most prominent concerns relate to the incidence of multiple pregnancies with ART treatment and the sub- 
sequent perinatal complications, including low birth weight and prematurity. ${ }^{10-12}$ Increasing the availability of integrated primary methods for infertility treatment that can be effectively provided by a trained generalist physician with low risk to women and offspring would potentially improve access to care for couples dealing with infertility.

Natural procreative technology (NaProTechnology, NPT) is an integrated and systematic approach to infertility that is suitable for primary care settings. It is based on a detailed study of events that occur during ovulation and throughout the menstrual cycle. ${ }^{13,14}$ Abnormalities of the reproductive cycle are identified and corrected to the extent possible. ${ }^{15-18}$ Patients receive thorough education about their fertility and are taught to monitor biomarkers of their own fertility cycles. In the process, they become equal partners in their own evaluation and treatment.

A standardized NPT investigation usually results in the diagnosis of one or more abnormalities of reproductive function that are associated with infertility. Abnormalities commonly identified include decreased production of estrogenic cervical mucus, intermenstrual bleeding or spotting, short or variable luteal phases, and suboptimal levels of the ovarian hormones estrogen and progesterone. ${ }^{19}$ The physician trained in NPT then determines a course of treatment that aims to correct the underlying abnormality, with the goal of optimizing physiologic conditions for conception in vivo. Common interventions include induction or stimulation of ovulation ${ }^{20}$; medications to enhance cervical mucus production, including vitamin B6, guaifenesin, or one of several antibiotics ${ }^{21,22}$; and hormonal supplementation in the luteal phase. ${ }^{23}$ Doses of all medications are adjusted according to the response of biomarkers and serum levels of estrogen and progesterone measured in the midluteal phase. ${ }^{13,17}$ Concurrently, couples use their awareness of ovulation biomarkers to time acts of intercourse to maximize chances of conception. ${ }^{24}$ Ongoing evaluation and support during pregnancy often includes supplementation with human-identical progesterone, based on periodic measurement of progesterone levels, in an effort to reduce the risk of adverse pregnancy outcomes. ${ }^{25-28}$

Outcomes for NPT have been published previously for a cohort of infertility patients in a specialty practice at its place of development (Creighton University). ${ }^{29}$ This study was undertaken to assess the outcomes of NPT as applied by trained generalist physicians in Galway, Ireland. Although NPT has been developed with both medical and surgical protocols, this study evaluates the medical protocols. We focused on the clinically relevant outcomes of live birth and multiple births.

\section{Methods}

During the study period, the clinic was a single physician office, with the exception of January 2000 to August 2001, when a second physician was practicing in the same office. The 2 physicians were licensed family physicians in Ireland who had received additional training in NPT through Creighton University (Omaha, Nebraska) and had successfully passed all evaluations and examinations of the NPT continuing medical education course. The clinic primarily focuses on providing women's health services.

Data for the NPT treatment cohort were collected from patients during their initial assessment visit and at subsequent follow-up visits and recorded in the routine medical record for the practice. Data from routine follow-up telephone contacts were also included in the medical records. The relevant data for this study were abstracted from medical records and entered into a computerized database, with manual verification of all entered data.

Patients were primarily self-referred couples experiencing difficulty conceiving and achieving a live birth. All patients from Ireland who presented between February 1998 and January 2002 (inclusive) and who proceeded beyond the initial explanatory consultation were eligible for inclusion in this study. Patients who had previously attempted ART (including in vitro fertilization and intracytoplasmic sperm injection) were also included. A few patients were advised after the initial consultation that they were not eligible for NPT, mostly because of azoospermia or menopause; these patients were not included in this analysis. We also excluded patients that had been trying for less than a year or who did not continue with the evaluation that was recommended at the initial consultation.

The NPT infertility treatment implemented in this clinic is a systematic multilevel investigation and treatment program. ${ }^{13}$ It begins with an initial consultation in which reproductive physiology and the various stages of NPT investigation and treat- 
ment are explained in detail to patients. They next learn specialized fertility tracking of daily observations of vaginal discharge biomarkers (bleeding and cervical fluid), according to the Creighton Model FertilityCare System. ${ }^{15,16,30}$ After patients gained competence in fertility tracking (no earlier than the second month), timed blood samples were taken with respect to the estimated time of ovulation as prospectively identified by the woman's own fertility chart. ${ }^{13,31}$ Reproductive hormones, including estradiol and progesterone, were measured and interpreted in the context of the woman's fertility tracking chart. Medications to correct identified hormonal abnormalities were prescribed after the completion of these investigations, usually in the fourth month. The goal was to optimize physiologic conditions for natural conception in each menstrual cycle, including mucus discharge quality, luteal hormone levels, ovulation, and the timing of intercourse. It often takes several cycles of treatment to reach optimal physiologic conditions. Once reached, there may still be some suboptimal cycles mixed with the optimal ones; for example, a cycle where intercourse does not occur during the fertile time. In addition, some patients conceive and subsequently experience a spontaneous abortion, after which they continue in the treatment program. Patients were advised that a total of up to 24 months may be required to complete an adequate trial of NPT to achieve pregnancy leading to a live birth. Evaluation and treatment of the male partner also took place during this time, as indicated. Hormonal assessment and support continued through pregnancy, as indicated..$^{25}$ Although specialized NPT surgical treatment may also form part of the NPT program, it was not available in this clinic in this time frame. A more detailed discussion of the NPT approach to infertility is available elsewhere. ${ }^{13,19,32}$

Per-cycle pregnancy rates are not an appropriate outcome to assess for NPT because of the longitudinal and incremental nature of the NPT treatment program. ${ }^{33,34}$ Therefore, we chose a cohort approach with the couple, rather than the cycle, as the unit of analysis. The main analyses for this study were the cumulative proportion of couples experiencing a first live birth during discrete time periods after the beginning of treatment. For comparability with other reports of infertility outcomes, we assigned the time of this outcome as the time of conception leading to first live birth. ${ }^{35}$ Secondarily, we also analyzed the cumulative proportion of first conceptions and cumulative proportion of withdrawals. Life-table analysis was used to adjust for couples withdrawing from treatment, or that completed 24 months of NPT treatment without pregnancy. Among the live births, we analyzed the proportion of multiple births. Secondarily, we analyzed the proportion with low birth weight and prematurity.

The study protocol was reviewed and approved by the Linacre Centre for Health care Ethics in London, and the Institutional Review Board for Human Subjects at the University of Utah. Because data were abstracted from usual clinical data sources and patient anonymity was maintained, there was no requirement for written informed consent of participants.

\section{Results}

A total of 1239 couples obtained an initial consult for NPT during the study period. Of these, 167 couples were excluded because they had tried for less than a year to conceive or because they did not continue evaluation beyond the initial consultation. The final study cohort consisted of 1072 couples that began treatment between February 1998 and January 2002 (inclusive), with subsequent outcomes followed through February 1, 2008. The women were, on average, 35.8 years old (range, 25 to 48 years), and nearly all were white. One third (33\%) had previously attempted ART treatment. The mean length of time that couples had attempted to conceive before NPT treatment was 5.6 years (range, 1 to 20 years). Approximately one fourth (24\%) of couples had a previous live birth. As shown in Table 1, the 364 couples who ultimately conceived with NPT treatment were slightly younger (mean age, 34.8 years); had not been attempting conception as long (mean duration, 4.8 years); were likely to have had a previous birth (30\%); and less likely to have attempted ART treatment $(21 \%)$.

In addition to teaching women to track their fertility biomarkers with the Creighton Model instruction, the most common diagnoses given to couples before and after NPT evaluation are shown in Table 2. Through NPT evaluation, more than half of the couples had suboptimal serum levels of progesterone and estradiol during the luteal phase. Approximately one fourth had limited cervical mucus and $10 \%$ had polycystic ovarian syndrome. 
Table 1. Characteristics of Couples Beginning Treatment with Natural Procreative Technology, by Subsequent Conception Status

\begin{tabular}{lccc}
\hline Patient Characteristic & $\begin{array}{c}\text { All } \\
\text { Eligible Couples* }\end{array}$ & $\begin{array}{c}\text { NPT Treatment, } \\
\text { Conceived }\end{array}$ & $\begin{array}{c}\text { NPT Treatment, } \\
\text { Did Not Conceive }\end{array}$ \\
\hline Total (n) ${ }^{\dagger}$ & 1072 & 364 & 708 \\
Woman's age (mean years [range]) & $35.8(25-48)$ & $34.8(25-45)$ & $36.4(26-48)$ \\
Prior years attempting to conceive (mean & $5.6(1-20)$ & $4.8(1-17)$ & $6.1(1-20)$ \\
$\quad[$ range]) & 24 & 30 & 20 \\
Had previous live birth (percent yes) & 33 & 21 & 39 \\
Received previous ART ${ }^{\ddagger}$ (percent yes) & & \\
\hline
\end{tabular}

*One hundred sixty-seven couples were not eligible because they had been trying for less than 1 year or because they did not complete the evaluation after the initial consultation.

${ }^{\dagger}$ Number of couples in each category. Age was available for all women. For previous years attempting to conceive, 30 (2.8\%) had missing data; for previous births, 30 (2.8\%) had missing data; for previous ART, 20 (1.9\%) had missing data.

${ }^{\ddagger}$ Assisted reproductive technology (ART) includes in vitro fertilization with or without intracytoplasmic sperm injection.

The most common treatments given to women included clomiphene (75.3\%), support of luteal hormonal production with human chorionic gonadotropin $(67 \%)$ or progesterone $(18 \%)$, and medications to enhance cervical mucus production (71\%). Fifty-four women (5\%) conceived without medical intervention, using only Creighton Model fertility charting and optimally timed intercourse.

There were 354 clinically recognized conceptions within 24 months after starting NPT treatment, with cumulative crude proportions of live births of 19.1 per 100 couples up to 12 months, and 25.5 up to 24 months, as shown in Table 3. Adjusting for withdrawals from treatment and continuing treatment at the end of study follow-up, the cumulative proportion of first live births was 27.1 up to 12 months, and 52.8 at 24 months. The proportions with any conception (regardless of its out- come) were higher: 25.9 crude and 35.5 adjusted at 12 months, and 33.0 crude and 64.8 adjusted at 24 months. Of conceptions within 2 years leading to a live birth, $75 \%$ (205 of 273 ) occurred within 12 months and 93\% (255 of 273) occurred within 18 months.

Several couple characteristics were associated with the probability of live birth, as shown in Table 4. The cumulative proportion of live births declined with increasing age of the woman. For women under 30, the crude proportion of live birth after 24 months was 33.7. For women over 40 the crude proportion was 13.9. The cumulative crude proportion of live birth also declined with increasing previous attempts to conceive (36.6 for previous time of 1 to 3 years; 11.9 for previous time more than 9 years), and with the number of previous ART attempts (30.8 for none, and 10.3 for 3 or

Table 2. Common Diagnoses of Couples Receiving Treatment Before and After Evaluation with Natural Procreative Technology*

\begin{tabular}{lcc}
\hline Diagnostic Category & $\begin{array}{c}\text { Before NPT Evaluation } \\
\text { (n [\%]) }\end{array}$ & $\begin{array}{c}\text { After NPT Evaluation } \\
\text { (n [\%] })\end{array}$ \\
\hline Unexplained infertility & $506(47.2)$ & $5(0.5)$ \\
Unexplained recurrent miscarriage & $124(11.6)$ & $2(0.2)$ \\
Anovulation & $31(2.9)$ & $36(3.4)$ \\
Polycystic ovarian syndrome & $68(6.3)$ & $110(10.3)$ \\
Endometriosis & $209(19.5)$ & $208(24.6)$ \\
Male factor & $115(10.7)$ & $146(13.6)$ \\
Limited cervical mucus & $12(1.1)$ & $276(25.7)$ \\
Suboptimal luteal progesterone & $99(9.2)$ & $923(86.1)$ \\
Suboptimal luteal estrogen & $2(0.2)$ & $676(63.1)$ \\
\hline
\end{tabular}

*This table is based on the 1072 couples that initiated evaluation. Diagnostic categories sum to more than $100 \%$ because couples could have more than one diagnosis (other than unexplained). 
Table 3. Cumulative Outcomes per 100 Couples by Time Completed in Natural Procreative Technology Evaluation and Treatment

\begin{tabular}{|c|c|c|c|c|c|c|c|c|c|}
\hline \multirow[b]{2}{*}{$\begin{array}{l}\text { Time Interval } \\
\text { (months) }\end{array}$} & \multirow[b]{2}{*}{$\begin{array}{c}\text { Cumulative } \\
\text { Withdrawals } \\
\text { from } \\
\text { NPT (n } \\
\text { "proportion") }\end{array}$} & \multicolumn{4}{|c|}{ Conceptions } & \multicolumn{4}{|c|}{ Live Births* } \\
\hline & & $\begin{array}{c}\text { Starting } \\
\text { at Time } \\
\text { Interval } \\
\text { (n) }\end{array}$ & $\begin{array}{l}\text { Cumulative } \\
\text { Conceptions } \\
\text { (n) }\end{array}$ & $\begin{array}{c}\text { Crude } \\
\text { Proportion }\end{array}$ & $\begin{array}{c}\text { Adjusted } \\
\text { Proportion }^{\dagger}\end{array}$ & $\begin{array}{c}\text { Starting at } \\
\text { Time } \\
\text { Interval } \\
\text { (n) }\end{array}$ & $\begin{array}{l}\text { Cumulative } \\
\text { Live Births } \\
\text { (n) }\end{array}$ & $\begin{array}{c}\text { Crude } \\
\text { Proportion }\end{array}$ & $\begin{array}{c}\text { Adjusted } \\
\text { Proportion }\end{array}$ \\
\hline $0-3$ & $105(9.8)$ & 1072 & 75 & 7.0 & 7.3 & 1072 & 55 & 5.1 & 5.4 \\
\hline $4-6$ & $233(21.7)$ & 892 & 152 & 14.2 & 15.9 & 895 & 111 & 10.4 & 11.8 \\
\hline $7-12$ & 478 (44.6) & 687 & 278 & 25.9 & 35.5 & 694 & 205 & 19.1 & 27.1 \\
\hline $13-18$ & $624(58.2)$ & 316 & 326 & 30.4 & 48.5 & 337 & 255 & 23.8 & 41.8 \\
\hline $19-24$ & $672(62.7)$ & 122 & 354 & 33.0 & 64.8 & 132 & 273 & 25.5 & 52.8 \\
\hline $25-36^{\ddagger}$ & & 46 & 364 & & & 56 & 286 & & \\
\hline
\end{tabular}

*Live births are assigned the time interval when the conception occurred rather than when the birth occurred.

${ }^{\dagger}$ Adjusted by life-table analysis, where withdrawal or continuing treatment at the end of study follow-up are censoring events.

${ }^{\ddagger}$ Proportions are not calculated beyond 24 months.

more). Women with a previous birth had a higher cumulative crude proportion of live birth (35.8) than those without (23.1). The adjusted proportions confirm the same trends but should not be considered reliable as precise estimates because most of the subgroup life table analyses involve small numbers of women continuing treatment until 24 months (ie, fewer than 25 women).

Among all live births observed, there were 13 twin births (4.6\%) and no higher order births. At least $88 \%$ of all births were to term and did not have low birth weight (Table 5). None of the pa-

Table 4. Live Births per 100 Couples at 24 months of Natural Procreative Technology Treatment by Characteristics of Couples Beginning Treatment

\begin{tabular}{|c|c|c|c|c|}
\hline Couple Category & Couples (n) & Live Births (n) & Crude Proportion & Adjusted Proportion* \\
\hline All couples & 1072 & 273 & 25.5 & 52.8 \\
\hline \multicolumn{5}{|c|}{ Woman's age (years) } \\
\hline$\leq 30$ & 86 & 29 & 33.7 & 59.1 \\
\hline$>30-35$ & 412 & 134 & 32.5 & 58.6 \\
\hline$>35-40$ & 423 & 89 & 21.0 & 46.1 \\
\hline$>40$ & 151 & 21 & 13.9 & 50.9 \\
\hline \multicolumn{5}{|c|}{ Time spent attempting to conceive (years) } \\
\hline $1-3$ & 246 & 90 & 36.6 & 66.0 \\
\hline$>3-6$ & 468 & 129 & 27.6 & 55.4 \\
\hline$>6-9$ & 210 & 39 & 18.6 & 44.9 \\
\hline$>9$ & 118 & 14 & 11.9 & 42.8 \\
\hline \multicolumn{5}{|l|}{ Previous live birth } \\
\hline Yes & 257 & 92 & 35.8 & 73.9 \\
\hline No & 785 & 181 & 23.1 & 48.5 \\
\hline \multicolumn{5}{|c|}{ Previous ART attempts $(\mathrm{n})^{\dagger}$} \\
\hline 0 & 702 & 216 & 30.8 & 61.5 \\
\hline 1 & 128 & 29 & 22.7 & 41.9 \\
\hline 2 & 125 & 18 & 14.4 & 34.9 \\
\hline$\geq 3$ & 97 & 10 & 10.3 & 19.8 \\
\hline
\end{tabular}

*Adjusted by life-table analysis, where withdrawal or continuing treatment at the end of study follow-up are censoring events. Adjusted proportions should be interpreted with caution because of small numbers in subgroups.

${ }^{+}$Assisted Reproductive Technology (ART) includes in vitro fertilization (IVF) with or without intracytoplasmic sperm injection (ICSI). 
Table 5. Outcomes for Natural Procreative Technology Live Births $(\mathrm{n}=\mathbf{2 8 6})$

\begin{tabular}{lc}
\hline Outcome & $\mathrm{n}(\%)$ \\
\hline Multiple gestation & $13(4.5)$ \\
Gestational age (weeks) & \\
$\geq 37$ & $246(86.0)$ \\
$<37$ & $15(5.2)$ \\
Unknown & $25(8.7)$ \\
Birth weight (grams) & \\
$\geq 2500$ & $245(85.6)$ \\
$1500-2500$ & $13(4.5)$ \\
$<1500$ & $4(1.4)$ \\
Unknown & $24(8.4)$ \\
\hline
\end{tabular}

tients in this cohort experienced ovarian hyperstimulation syndrome. The proportion of couples withdrawing from treatment was 44.6 per 100 couples by 12 months, and 62.7 by 24 months (Table 1). Among couples who had a live birth, 51 couples had additional NPT treatment, resulting in 47 additional live births within the study period. However, the results reported here are either for the first conceptions or the first live births only.

\section{Discussion}

In this study setting, NPT, an integrative approach to infertility provided by NPT-trained family physicians, resulted in substantial live birth rates with a minimal risk of twin or multiple births. These results would suggest that NPT provided by trained generalist physicians has the potential to improve access to treatment for infertility with favorable outcomes. The question remains, however, as to how to interpret these results in relation to outcomes from other infertility treatments.

We reported the outcome of conception because this is an intermediate outcome of interest to patients and because it is an outcome commonly reported in infertility literature. However, we focused on live birth as the major outcome for this study because live birth is the outcome that is most clinically meaningful for patients seeking treatment for infertility. ${ }^{36,37}$ Crude birth rates substantially underestimate the efficacy of treatment because of high drop-out rates that are found in most studies of infertility treatment; however, life-table analysis overestimates treatment efficacy because it assumes that those discontinuing treatment have the same prognosis as those continuing treatment, an as- sumption that may not be valid for infertility treatment. ${ }^{38,39}$ Thus, the "true" estimates for NPT success probably lie somewhere between the adjusted life-table estimates and the crude estimates that we have reported.

We believe our study can be considered to represent a "real world" effectiveness based on undifferentiated infertility categories because all patients who were eligible for treatment were included, as would normally occur in clinical practice. However, the population presenting in this study for NPT treatment may represent a more difficult group than would be normally seen even in some specialist ART clinics; $33 \%$ of those presenting for NPT treatment had previously attempted ART. In addition, the mean duration of time spent previously trying to conceive for this population was 5.6 years. Finally, this was a relatively older population of women, with a mean age at entry to treatment of just over 35 years. Each of these characteristics is a risk factor strongly associated with lower likelihood of conception. ${ }^{40-44}$ In comparison, a recent national Dutch cohort of 4928 women undergoing ART had an average age of 33.6 years and a mean duration of attempting conception of 3.6 years, with a 1-year cumulative probability of ongoing pregnancy of $45 \% .{ }^{35}$ Importantly, the duration of time attempting conception in our study is greater than of all the studies with which we compare in the remaining discussion, and the mean age of the women in our study is also greater than most of them.

Comparison of these results to studies of other infertility treatments must be made tentatively, because results vary by location, diagnosis, and both identified and unidentified risk factors. ${ }^{45,46}$ Unfortunately, most data about infertility treatment outcomes are reported on a per-cycle basis, so pregnancy or birth rates may be biased by high rates of discontinuation of treatment, especially for cohorts that have liberal entrance criteria. ${ }^{33,38}$ However, some studies of infertility have used a cohort approach and can provide some benchmarks against which to evaluate our results, as detailed below.

Two cohort studies have demonstrated substantial rates of spontaneous conception in some patients with infertility, including a population-based study with a live birth rate of $40 \%{ }^{40}$ and a referral clinic population with a live birth rate of $20 \%,{ }^{41}$ both over 24 months. Both studies, however, included women with an average age of 29 and an 
average infertility duration of 2 and 3.5 years, respectively. Thus, the prognosis for spontaneous conception in these studies was substantially higher than for the patients in our study. None of the patients in the spontaneous conception studies had previously attempted ART, whereas $33 \%$ of the couples in our study had done so.

Another comparison of our results would be with population-based studies of infertility treatment. In a 1985 quasi population-based study, there was an overall conception rate of 48 per 100 couples for all causes of infertility after 2 years of treatment, adjusted by life-table analysis (treatments included ovulation induction, artificial insemination, hormonal treatment, and/or surgery). ${ }^{44}$ Again, the population in this study was much younger (women's mean age, 28 years) than our study; had a much lower duration of infertility before treatment (mean duration, 2.4 years); and excluded couples that had previously attempted ART. Closer to comparison with our study, the subgroup of women with unexplained infertility of 5 or more years' duration had a 2 -year adjusted pregnancy rate of approximately $30 \%$, compared with $53 \%$ in our study.

How do these results compare to existing data for ART? The Human Fertilisation and Embryology Authority's crude live birth rate for the year 2000 was 23.8 per 100 women, ${ }^{47}$ which is comparable to the crude live births of 22.7 in this study; but our results are for a longer time frame of up to 2 years. In the United States in 2000, registry data for ART indicated a $25.4 \%$ live birth rate per ART cycle initiated (excluding donor eggs or frozen embryos), but no data are available on the number of women treated or follow-up over time. ${ }^{37,48} \mathrm{Al}-$ though more recent ART data are available, we have referenced 2000 data for comparability to the time frame during which the patients in this study were treated. Given the differences in time frames, the fact that women's characteristics and longitudinal outcomes cannot be extracted from registry data, and other dissimilarities, this comparison must only be exploratory.

Although life-table analysis is not possible with the existing registry data for ART, other studies have reported cumulative pregnancy or live birth rates in cohorts with multiple attempts at ART. These results varied from a low $32 \%{ }^{49}$ to a range of $39 \%$ to $60 \%,{ }^{38,50}$ to a high of $64.7 \% .{ }^{39}$ In this last study the mean age of women was 32.8 years, the mean duration of infertility 3.6 years, and no couples had previously failed ART; all of this suggests that the patients studied had a better prognosis than patients in our study. In a recent populationbased cohort of couples receiving ART in the Netherlands, the cumulative pregnancy rate at 1 year was $45 \%$. ${ }^{35}$ Taken together, these results suggest that the overall success rates of treatment with NPT might be comparable to ART despite greater per-cycle pregnancy rates with ART. Although it may seem paradoxical that a treatment with a lower per-cycle pregnancy rate than ART may have a comparable cumulative rates of pregnancy or live birth, very similar results have also been found in randomized trials of ART versus other less invasive therapies for couples with unexplained infertility. ${ }^{51,52}$ A systematic Cochrane review concluded that there are insufficient data to establish that ART is any more effective than less intensive therapies for unexplained infertility. ${ }^{53}$

Multiple births are one of the most notable and important complications of ART as it is currently practiced. ${ }^{6,54}$ The proportion of live births of multiples was much lower in this NPT cohort (4.6\%) than for ART treatment in the UK in 1998 to 1999 (27.1\%). ${ }^{47,48}$ Although multiple birth rates from ART have dropped somewhat, they remain high in most settings, eg, $34 \%$ in the US in $2003 .{ }^{37,48}$ Longitudinal studies of long-term outcomes after NPT treatment are necessary to determine whether NPT treatment might have fewer longterm health risks for resulting children than ART.

A weakness of our study is that over half of patients withdrew from treatment before completing a full 24-month course of treatment. In comparison, in cohort studies of ART of up to 5 cycles in which cost was not an issue, dropout rates from treatment were $69 \%$ and $55 \%,{ }^{38,39}$ and in a randomized trial of ART versus other treatments, the overall dropout rate was $45 \% .{ }^{51}$ Thus, the dropout rates from treatment in our study are comparable to those of studies of other fertility treatments. Withdrawing from treatment affects pregnancy or live birth probabilities adjusted by life-table analysis but does not change the crude probabilities that we have reported for all outcomes. Crude probabilities include all couples in the analysis, treating those that dropped out of treatment as if they continued treatment, and therefore represent the most conservative estimate of treatment outcomes. Future studies should seek to minimize the withdrawal 
rates to the extent possible and to define the prognostic factors among those withdrawing to estimate the differential effect of dropout on adjusted probabilities of pregnancy or live birth.

We emphasize again that comparisons of the results of this study with other available data, as discussed above, are limited by many differences in methods and study populations. More recent studies of ART tend to show slight improvement of pregnancy rates in European countries over the past few years, where there is a strong tendency to transfer fewer embryos, and more substantial improvement in pregnancy rates in the United States, where multiple gestations also remain substantially more frequent than in Europe. ${ }^{55,56}$ It remains to be seen whether NPT pregnancy rates may improve with time as well.

\section{Conclusion}

This study demonstrates that NPT is an effective integrated system of infertility treatment that can be done by interested and appropriately trained generalist physicians. The cumulative crude pregnancy rates, withdrawal rates, and adjusted pregnancy rates are similar to cohort studies of ART based on the limited comparable data that are available. The treatment program is minimally invasive, with fewer multiple pregnancies. However, it is not an option for couples with azoospermia, ovarian failure, or bilateral fallopian tube occlusion. The probability of live birth with NPT treatment is, as expected, lower for women over age 35, couples who have spent more than 6 years trying to conceive, couples without previous births, and couples who have previously attempted ART; these are risk factors that are also associated with lower live birth rates with other types of fertility treatments. ${ }^{44}$ The most significant risk factor for decreased live birth with ART is increased maternal age. ${ }^{35}$ Couples choosing NPT need to clearly understand that, although $75 \%$ couples that have a live birth with NPT conceive within 12 months (93\% within 18 months), it may take up to 24 months for a full treatment course. For women who are approaching the end of their reproductive years, the time frame of up to 2 years required for NPT treatment may be a significant disadvantage of NPT.

Large multicenter prospective studies are warranted to confirm these results, to explore further the characteristics associated with successful NPT treatment, and to directly compare NPT to other forms of infertility treatment.

\section{References}

1. National Collaborating Centre for Women's and Children's Health (United Kingdom). Fertility: assessment and treatment for people with fertility problems. In: National Institute for Clinical Excellence. London: Royal College of Obstetricians and Gynaecologists Press; 2004.

2. Hull MG. Infertility treatment: relative effectiveness of conventional and assisted conception methods. Hum Reprod 1992;7:785-96.

3. Copperman AB, DeCherney AH. Turn, turn, turn. Fertil Steril 2006;85:12-3.

4. Govaerts I, Devreker F, Delbaere A, Revelard P, Englert Y. Short-term medical complications of 1500 oocyte retrievals for in vitro fertilization and embryo transfer. Eur J Obstet Gynecol Reprod Biol 1998;77:239-43.

5. Brinsden PR, Wada I, Tan SL, Balen A, Jacobs HS. Diagnosis, prevention and management of ovarian hyperstimulation syndrome. Br J Obstet Gynaecol 1995;102:767-72.

6. Schieve LA, Meikle SF, Ferre C, Peterson HB, Jeng $\mathrm{G}$, Wilcox LS. Low and very low birth weight in infants conceived with use of assisted reproductive technology. N Engl J Med 2002;346:731-7.

7. Hansen M, Kurinczuk JJ, Bower C, Webb S. The risk of major birth defects after intracytoplasmic sperm injection and in vitro fertilization. N Engl J Med 2002;346:725-30.

8. Stromberg B, Dahlquist G, Ericson A, Finnstrom O, Koster M, Stjernqvist K. Neurological sequelae in children born after in-vitro fertilisation: a population-based study. Lancet 2002;359:461-5.

9. Mitchell AA. Infertility treatment-more risks and challenges. N Engl J Med 2002;346:769-70.

10. Multiple gestation pregnancy. The ESHRE Capri Workshop Group. Hum Reprod 2000;15:1856-64.

11. Schieve LA, Peterson HB, Meikle SF, et al. Livebirth rates and multiple-birth risk using in vitro fertilization. JAMA 1999;282:1832-8.

12. Kissin DM, Schieve LA, Reynolds MA. Multiplebirth risk associated with IVF and extended embryo culture: USA, 2001. Hum Reprod 2005;20:2215-23.

13. Boyle PC. NaPro technology and infertility: a family physician's approach. In: Hilgers TW, ed. The medical and surgical practice of NaProTechnology. Omaha (NE): Pope Paul VI Institute Press; 2004: 653-66.

14. Hilgers TW. What is NaProTechnology? In: Hilgers TW, ed. The medical and surgical practice of NaProTechnology. Omaha (NE): Pope Paul VI Institute Press; 2004:19-28.

15. Hilgers TW, Daly KD, Hilgers SK, Prebil AM. Creighton Model Fertility Care System: a standard- 
ized, case management appproach to teaching, book 1, 2nd ed. Omaha (NE): Pope Paul VI Institute Press; 2002.

16. Hilgers TW. Introduction to the Creighton Model System. In: Hilgers TW, ed. The medical and surgical practice of NaProTechnology. Omaha (NE): Pope Paul VI Institute Press; 2004:43-56.

17. Hilgers TW. Medical treatment of ovarian and target organ dysfunction. In: Hilgers TW, ed. The medical and surgical practice of NaProTechnology. Omaha (NE): Pope Paul VI Institute Press; 2004: 607-34.

18. Hilgers TW. Summary of NaPro technology biomarkers. In: Hilgers TW, ed. The medical and surgical practice of NaProTechnology. Omaha (NE): Pope Paul VI Institute Press; 2004:1137-62.

19. Hilgers TW. NaPro technology in infertility: evaluation and treatment. In: Hilgers TW, ed. The medical and surgical practice of NaProTechnology. Omaha (NE): Pope Paul VI Institute Press; 2004: 509-40.

20. The Practice Committee of the American Society for Reproductive Medicine. Use of clomiphene in women. Fertil Steril 2003 80:1302-8.

21. Check JH, Adelson HG, Wu CH. Improvement of cervical factor with guaifenesin. Fertil Steril 1982;37: 707-8.

22. Check JH. Diagnosis and treatment of cervical mucus abnormalities. Clin Exp Obstet Gynecol 2006; 33:140-2.

23. Pritts EA, Atwood AK. Luteal phase support in infertility treatment: a meta-analysis of the randomized trials. Hum Reprod 2002;17:2287-99.

24. Stanford JB, Smith KR, Dunson DB. Vulvar mucus observations and the probability of pregnancy. Obstet Gynecol 2003;101:1285-93.

25. Hilgers TW. Using progesterone support during pregnancy. In: Hilgers TW, ed. The medical and surgical practice of NaProTechnology. Omaha (NE): Pope Paul VI Institute Press; 2004:725-46.

26. Hilgers TW. Assessing progesterone during pregnancy. In: Hilgers TW, ed. The medical and surgical practice of NaProTechnology. Omaha (NE): Pope Paul VI Institute Press; 2004:713-24.

27. Meis PJ, Klebanoff $M$, Thom E, et al. Prevention of recurrent preterm delivery by 17 alpha-hydroxyprogesterone caproate. N Engl J Med 2003;348:237985.

28. da Fonseca EB, Bittar RE, Carvalho MH, Zugaib M. Prophylactic administration of progesterone by vaginal suppository to reduce the incidence of spontaneous preterm birth in women at increased risk: a randomized placebo-controlled double-blind study. Am J Obstet Gynecol 2003;188:419-24.

29. Hilgers TW. Effectiveness of NaPro technology in the treatment of infertility. In: Hilgers TW, ed. The medical and surgical practice of NaProTechnology.
Omaha (NE): Pope Paul VI Institute Press; 2004: 677-94.

30. Hilgers T. The Creighton Model FertilityCare System: an introductory booklet for new users, 5 th ed. Omaha (NE): Pope Paul VI Institute Press; 2001.

31. Hilgers TW. Targeted hormone assessment of the menstrual cycle. In: Hilgers TW, ed. The medical and surgical practice of NaProTechnology. Omaha (NE): Pope Paul VI Institute Press; 2004:251-8.

32. Danis P. A family physician's experience with NaPro technology. In: Hilgers TW, ed. The medical and surgical practice of NaProTechnology. Omaha (NE): Pope Paul VI Institute Press; 2004:1101-14.

33. Daya S. Pitfalls in the design and analysis of efficacy trials in subfertility. Hum Reprod 2003;18:1005-9.

34. Vail A, Gardener L. Reply to Dickey: clinical as well as statistical knowledge. Hum Reprod 2003; 18:2495-8.

35. Lintsen AM, Eijkemans MJ, Hunault CC, et al. Predicting ongoing pregnancy chances after IVF and ICSI: a national prospective study. Hum Reprod 2007;22:2455-62.

36. Thurin A, Hausken J, Hillensjo T, et al. Elective single-embryo transfer versus double-embryo transfer in in vitro fertilization. N Engl J Med 2004;351: 2392-402.

37. Centers for Disease Control and Prevention. Assisted reproductive technology success rates 2003 . National summary and fertility clinic reports. Washington, DC: US Department of Health and Human Services; 2003.

38. Stolwijk AM, Hamilton CJ, Hollanders JM, Bastiaans LA, Zielhuis GA. A more realistic approach to the cumulative pregnancy rate after in-vitro fertilization. Hum Reprod 1996;11:660-3.

39. Stolwijk AM, Wetzels AM, Braat DD. Cumulative probability of achieving an ongoing pregnancy after in-vitro fertilization and intracytoplasmic sperm injection according to a woman's age, subfertility diagnosis and primary or secondary subfertility. Hum Reprod 2000;15:203-9.

40. Snick HK, Snick TS, Evers JL, Collins JA. The spontaneous pregnancy prognosis in untreated subfertile couples: the Walcheren primary care study. Hum Reprod 1997;12:1582-8.

41. Collins JA, Burrows EA, Wilan AR. The prognosis for live birth among untreated infertile couples. Fertil Steril 1995;64:22-8.

42. Eimers JM, te Velde ER, Gerritse R, Vogelzang ET, Looman CW, Habbema JD. The prediction of the chance to conceive in subfertile couples. Fertil Steril 1994;61:44-52.

43. Dunson DB, Baird DD, Colombo B. Increased infertility with age in men and women. Obstet Gynecol 2004;103:51-6.

44. Hull MG, Glazener CM, Kelly NJ, et al. Population study of causes, treatment, and outcome of infertility. Br Med J (Clin Res Ed) 1985;291:1693-7. 
45. Juul S, Karmaus W, Olsen J. Regional differences in waiting time to pregnancy: pregnancy-based surveys from Denmark. France, Germany, Italy and Sweden. The European Infertility and Subfecundity Study Group. Hum Reprod 1999;14:1250-4.

46. Land JA, Evers JL. What is the most relevant standard of success in assisted reproduction? Defining outcome in ART: a Gordian knot of safety, efficacy and quality. Hum Reprod 2004;19:1046-8.

47. Human Fertilisation and Embryology Authority. Ninth annual report and accounts. London: Human Fertilisation and Embryology Authority; 2000.

48. Wright VC, Schieve LA, Reynolds MA, Jeng G. Assisted reproductive technology surveillanceUnited States, 2000. MMWR Surveill Summ 2003; 52:1-16.

49. Nargund G, Waterstone J, Bland J, Philips Z, Parsons J, Campbell S. Cumulative conception and live birth rates in natural (unstimulated) IVF cycles. Hum Reprod 2001;16:259-62.

50. Tan SL, Doyle P, Maconochie N, et al. Pregnancy and birth rates of live infants after in vitro fertilization in women with an without previous in vitro fertilization pregnancies: a study of eight thousand cycles at one center. Am J Obstet Gynecol 1994; 170(1 Pt 1):34-40.
51. Goverde AJ, McDonnell J, Vermeiden JP, Schats R, Rutten FF, Schoemaker J. Intrauterine insemination or in-vitro fertilisation in idiopathic subfertility and male subfertility: a randomised trial and cost-effectiveness analysis. Lancet 2000;355:13-8.

52. Johnson NP, Proctor M, Farquhar CM. Gaps in the evidence for fertility treatment-an analysis of the Cochrane Menstrual Disorders and Subfertility Group database. Hum Reprod 2003;18:947-54.

53. Pandian Z, Bhattacharya S, Vale L, Templeton A. In vitro fertilisation for unexplained subfertility. Cochrane Database Syst Rev 2005 ;(2):CD003357.

54. Olivennes F, Fanchin R, Ledee N, Righini C, Kadoch IJ, Frydman R. Perinatal outcome and developmental studies on children born after IVF. Hum Reprod Update 2002;8:117-28.

55. Wright VC, Chang J, Jeng G, Chen M, Macaluso M. Assisted reproductive technology surveillanceUnited States, 2004. MMWR Surveill Summ 2007; 56:1-22.

56. Andersen AN, Goossens V, Ferraretti AP, et al. Assisted reproductive technology in Europe, 2004: results generated from European registers by ESHRE. Hum Reprod 2008;23:756-71. 